Y. H. Lee

Nagoya Math. J.

Vol. 162 (2001), 149-167

\title{
SOLUTIONS OF \\ A CERTAIN NONLINEAR ELLIPTIC EQUATION ON RIEMANNIAN MANIFOLDS
}

\author{
YONG HAH LEE
}

\begin{abstract}
In this paper, we prove that if a complete Riemannian manifold $M$ has finitely many ends, each of which is a Harnack end, then the set of all energy finite bounded $\mathcal{A}$-harmonic functions on $M$ is one to one corresponding to $\mathbf{R}^{l}$, where $\mathcal{A}$ is a nonlinear elliptic operator of type $p$ on $M$ and $l$ is the number of $p$-nonparabolic ends of $M$. We also prove that if a complete Riemannian manifold $M$ is roughly isometric to a complete Riemannian manifold with finitely many ends, each of which satisfies the volume doubling condition, the Poincare inequality and the finite covering condition near infinity, then the set of all energy finite bounded $\mathcal{A}$-harmonic functions on $M$ is finite dimensional. This result generalizes those of Yau, of Donnelly, of Grigor'yan, of Li and Tam, of Holopainen, and of Kim and the present author, but with a barrier argument at infinity that the peculiarity of nonlinearity demands.
\end{abstract}

\section{$\S 1$. Introduction}

The classical Liouville theorem, which states that any bounded harmonic function on $\mathbf{R}^{2}$ must be constant, has long been an interesting topic of study to analysts and geometers. In 1975, Yau [20] proved a remarkable result that any complete Riemannian manifold with nonnegative Ricci curvature has no nonconstant positive harmonic functions. On the other hand, the validity of the Liouville property means that the space of positive harmonic functions on the manifold is one dimensional. In this view point, it is natural to regard the finite dimensionality of the space of positive harmonic functions as a generalized version of the Liouville property. Later, such a theory is, in this line, well developed by the works of Donnelly [6], Grigor'yan [7], Li and Tam [15], [16], Kim and the present author [14], and others.

In this paper, we consider, in line with the above viewpoint, the generalized version of the Liouville property for solutions for a nonlinear elliptic

Received November 29, 1999.

2000 Mathematics Subject Classification: 53C21; 31C12. 
operator $\mathcal{A}$ in the following setting: Let $M$ be a complete Riemannian manifold and $\Omega$ be an open subset of $M$. Let $W^{1, p}(\Omega)$ be the Sobolev space of all functions $u \in L^{p}(\Omega)$ whose distributional gradient $\nabla u$ also belongs to $L^{p}(\Omega)$. We equip $W^{1, p}(\Omega)$ with the norm $\|u\|_{1, p}=\|u\|_{p}+\|\nabla u\|_{p}$. The space $W_{0}^{1, p}(\Omega)$ is the closure of $C_{0}^{\infty}(\Omega)$ in $W^{1, p}(\Omega)$.

Let $T \Omega=\cup_{x \in \Omega} T_{x} M$. Suppose that a map $\mathcal{A}: T \Omega \rightarrow T \Omega$ satisfies the following assumptions for some constants $1<p<\infty$ and $0<C_{1} \leq C_{2}<$ $\infty$;

(A1) the mapping $\mathcal{A}_{x}=\left.\mathcal{A}\right|_{T_{x} M}: T_{x} M \rightarrow T_{x} M$ is continuous for a.e. $x \in \Omega$, and the mapping $x \mapsto \mathcal{A}_{x}(\xi)$ is a measurable vector field whenever $\xi$ is; for a.e. $x \in \Omega$ and for all $\xi \in T_{x} M$

$(\mathrm{A} 2)\left\langle\mathcal{A}_{x}(\xi), \xi\right\rangle \geq C_{1}|\xi|^{p}$

(A3) $\left|\mathcal{A}_{x}(\xi)\right| \leq C_{2}|\xi|^{p-1}$

(A4) $\left\langle\mathcal{A}_{x}\left(\xi_{1}\right)-\mathcal{A}_{x}\left(\xi_{2}\right), \xi_{1}-\xi_{2}\right\rangle>0$ whenever $\xi_{1} \neq \xi_{2}$;

(A5) $\mathcal{A}_{x}(\lambda \xi)=\lambda|\lambda|^{p-2} \mathcal{A}_{x}(\xi)$ whenever $\lambda \in \mathbf{R}, \lambda \neq 0$.

A function $u$ in $W_{\text {loc }}^{1, p}(\Omega)$ is a solution (supersolution, subsolution, respectively) of the equation

$$
-\operatorname{div} \mathcal{A}_{\mathrm{x}}(\nabla \mathrm{u})=0(\geq 0, \leq 0, \text { respectively })
$$

in $\Omega$ if

$$
\int_{\Omega}\left\langle\mathcal{A}_{x}(\nabla u), \nabla \phi\right\rangle=0(\geq 0, \leq 0, \text { respectively })
$$

for any (nonnegative, respectively) $\phi \in C_{0}^{\infty}(\Omega)$. We say that a function $u$ is $\mathcal{A}$-harmonic (of type $p$ ) if $u$ is a continuous solution of the equation (1). In the typical case $\mathcal{A}_{x}(\xi)=\xi|\xi|^{p-2}, \mathcal{A}$-harmonic functions are called $p$-harmonic and, in particular, if $p=2$, we obtain harmonic functions. An important property of $\mathcal{A}$-harmonic functions is the comparison principle as follows: if $u \in W^{1, p}(\Omega)$ is a supersolution and $v \in W^{1, p}(\Omega)$ is a subsolution of (1), respectively, in an open set $\Omega$, and $\min \{u-v, 0\} \in W_{0}^{1, p}(\Omega)$, then $u \geq v$ a.e. in $\Omega$. In particular, if both $u$ and $v$ are $\mathcal{A}$-harmonic in a bounded set $\Omega$ and $u \geq v$ on $\partial \Omega$, then $u \geq v$ in $\Omega$. (See [8].) A Green's function $G=G(x, \cdot)$ for the elliptic operator $\mathcal{A}$ on $M$ denotes (if exists) a positive solution of the equation

$$
-\operatorname{div} \mathcal{A}(\nabla \mathrm{G})=\delta_{\mathrm{x}}
$$


for each $x \in M$, in the sense of distributions, i.e.,

$$
\int_{M}\langle\mathcal{A}(\nabla G), \nabla \phi\rangle=\phi(x)
$$

for any $\phi \in C_{0}^{\infty}(M)$. It is known that a Green's function $G$ for the elliptic operator $\mathcal{A}$ on $M$ exists if and only if $M$ has positive $p$-capacity, i.e., there exists a compact subset $K \subset M$ such that

$$
\operatorname{Cap}_{p}(K, \infty, M)=\inf _{\phi} \int_{M}|\nabla \phi|^{p}>0,
$$

where the infimum is taken over all functions $\phi \in C_{0}^{\infty}(M)$ with $\phi=1$ on $K$. We say that a complete Riemannian manifold $M$ is $p$-parabolic if $M$ does not admit any Green's function $G$. Otherwise, $M$ is called $p$-nonparabolic.

Also, we consider functionals associated with $\mathbf{F}: T \Omega \rightarrow \mathbf{R}$, where

(A6) the mapping $\mathbf{F}_{x}=\left.\mathbf{F}\right|_{T_{x} M}: T_{x} M \rightarrow \mathbf{R}$ is strictly convex and differentiable for all $x \in \Omega$, and the mapping $x \mapsto \mathbf{F}_{x}(\xi)$ is measurable whenever $\xi$ is;

(A7) there exist constants $0<C_{1} \leq C_{2}<\infty$ such that

$$
C_{1}|\xi|^{p} \leq \mathbf{F}_{x}(\xi) \leq C_{2}|\xi|^{p}
$$

for all $x \in \Omega$ and $\xi \in T_{x} M$.

We will write

$$
\mathbf{J}(u, \Omega)=\int_{\Omega} \mathbf{F}_{x}(\nabla u) .
$$

Let $\mathcal{A}_{x}(\xi)=\left(\mathbf{A}^{1}(\xi), \mathbf{A}^{2}(\xi), \ldots, \mathbf{A}^{n}(\xi)\right)$ be defined by

$$
\mathbf{A}^{i}(\xi)=\frac{\partial}{\partial \xi^{i}} \mathbf{F}_{x}(\xi) \text { for } i=1,2, \ldots, n .
$$

Then $\mathcal{A}$ satisfies (A1) through (A4). In fact, given $f \in W_{0}^{1, p}(\Omega)$, each $\mathcal{A}$ harmonic function $h$ with $h-f \in W_{0}^{1, p}(\Omega)$ minimizes $\mathbf{J}$ in $\mathcal{H}=\{u \in$ $\left.W^{1, p}(\Omega): u-f \in W_{0}^{1, p}(\Omega)\right\}$. (See [18].) In the case when an $\mathcal{A}$-harmonic function $u$ on $M$ has finite energy, i.e., $\mathbf{J}(u, M)<\infty$, we say that $u$ is an energy finite $\mathcal{A}$-harmonic function. We now introduce an additional assumption on $\mathcal{F}$ as follows: For any $\xi_{1}, \xi_{2} \in T_{x} M$, 
(A8) in case $2 \leq p<\infty$

$$
\mathbf{F}_{x}\left(\frac{\xi_{1}+\xi_{2}}{2}\right)+\mathbf{F}_{x}\left(\frac{\xi_{1}-\xi_{2}}{2}\right) \leq \frac{1}{2}\left(\mathbf{F}_{x}\left(\xi_{1}\right)+\mathbf{F}_{x}\left(\xi_{2}\right)\right)
$$

in case $1<p \leq 2$,

$$
\mathbf{F}_{x}\left(\frac{\xi_{1}+\xi_{2}}{2}\right)^{p^{\prime}}+\mathbf{F}_{x}\left(\frac{\xi_{1}-\xi_{2}}{2}\right)^{p^{\prime}} \leq\left(\frac{1}{2}\left(\mathbf{F}_{x}\left(\xi_{1}\right)+\mathbf{F}_{x}\left(\xi_{2}\right)\right)\right)^{p^{\prime}}
$$

where $p^{\prime}=1 /(p-1)$.

In particular, using Clarkson's inequality, the assumption (A8) is satisfied in the typical case $\mathbf{F}(\xi)=\frac{1}{p}|\xi|^{p}$, i.e., $p$-harmonic case. (See [9].)

In the above setting, we can describe the set of nonnegative $\mathcal{A}$-harmonic functions on a complete Riemannian manifold $M$ in terms of ends of $M$ as follows:

THEOREM 1.1. Let $M$ be a complete Riemannian manifold with finitely many ends, each of which is a Harnack end. Let $E_{1}, E_{2}, \ldots, E_{l}$ and $e_{1}, e_{2}$, $\ldots, e_{s}$ be $p$-nonparabolic ends and p-parabolic ends, respectively, with $l \geq 1$ and $s \geq 0$. Then for any nonnegative $\mathcal{A}$-harmonic function $f$ on $M$, there exist real numbers $a_{1}, a_{2}, \ldots, a_{l} \in \mathbf{R}$ and a subset $P$ of $\{1,2, \ldots, s\}$ such that

$$
\begin{array}{r}
\lim _{x \rightarrow \infty, x \in E_{i}} f(x)=a_{i} ; \\
\lim _{x \rightarrow \infty, x \in e_{j}} f(x)=\infty,
\end{array}
$$

where $i=1,2, \ldots, l$ and $j \in P$. Conversely, given real numbers $a_{1}, a_{2}$, $\ldots, a_{l} \in \mathbf{R}$ and a subset $P$ of $\{1,2, \ldots, s\}$, there exists nonnegative $\mathcal{A}$ harmonic function $f$ satisfying (2) and (3).

Moreover, in the case when $\mathcal{A}$ satisfies (A5) through (A8), each energy finite bounded $\mathcal{A}$-harmonic function $f$ is uniquely determined by the values in $(2)$.

It is instructive to note that in the case of harmonic functions, we have only to construct generators of the space of positive harmonic functions. (See [7], [15], [16] and [14].) However in the above setting, there is no clear way to define generators due to the nonlinearity. Furthermore, a sum of $\mathcal{A}$-harmonic functions is not necessarily $\mathcal{A}$-harmonic again. Hence we need 
to directly construct each solution taking the given data at infinity of each end. The key argument in proving the existence theorem is to construct barriers at infinity corresponding to given data, which impose a solution to converge to given data at infinity, thereby we get the desired solution. On the other hand, the proof of the uniqueness is more delicate. In the case of the Laplacian, if a positive harmonic function converges asymptotically zero at infinity of each nonparabolic end, then by the maximum principle it is identically zero. Therefore, the uniqueness immediately follows from the linearity. However, in nonlinear case, this procedure is not feasible any longer. In Section 3, we introduce a rather subtle method, which depends deeply on the energy finiteness of solutions. This method, together with the barrier argument, plays a crucial role in proving the uniqueness in Theorem 1.1 .

That an end is Harnack means the validity of the Harnack inequality near infinity for nonnegative $\mathcal{A}$-harmonic functions on the end. Holopainen [10], [11] proved that the Harnack inequality forces each nonnegative $\mathcal{A}$ harmonic function to converge to a constant at infinity of each end, and gave various examples of Harnack ends. As another example of Harnack ends, we consider ends satisfying the volume doubling condition, the Poincaré inequality and the finite covering condition, expounded later. In fact, these conditions are valid on each end of a complete Riemannian manifold with nonnegative Ricci curvature outside a compact subset and finite first Betti number. (See [2], [3], [17] and [19].) An ingredient treated in this paper is the rough isometry, which is a more general one than the bi-Lipschitz map. We can prove the rough isometric invariance of the volume doubling condition, the Poincaré inequality and the finite covering condition, and hence we obtain that any end roughly isometric to an end satisfying these conditions is also Harnack. Moreover, the number of ends is invariant under rough isometries between complete Riemannian manifolds and each rough isometry between manifolds can be reduced to a rough isometry between ends. Also, following the program of Kanai [13], one can prove that the $p$-parabolicity is invariant under rough isometries between ends. (See also [10].) Hence, we get the same result as that of Theorem 1.1 on a lager class of complete Riemannian manifolds as follows:

Corollary 1.2. Let $M$ and $N$ be complete Riemannian manifolds satisfying the local volume doubling condition and the local Poincaré inequality, expounded later, and roughly isometric to each other. Suppose that 
$M$ has finitely many ends, each of which satisfies the volume doubling condition $(D)_{\infty}$, the Poincaré inequality $(P)_{\infty}$ and the finite covering condition (FC). Then $N$ has the same numbers of p-nonparabolic ends and p-parabolic ends as those of $M$, respectively, and each end of $N$ is Harnack.

Let $E_{1}, E_{2}, \ldots, E_{l}, l \geq 1$, and $e_{1}, e_{2}, \ldots, e_{s}, s \geq 0$, be p-nonparabolic ends and p-parabolic ends of $N$, respectively, and $\mathcal{A}$ be an elliptic operator on $N$ satisfying (A1) through (A5). Then for any nonnegative $\mathcal{A}$-harmonic function $f$ on $N$, there exist real numbers $a_{1}, a_{2}, \ldots, a_{l} \in \mathbf{R}$ and a subset $P$ of $\{1,2, \ldots, s\}$ such that

$$
\begin{aligned}
\lim _{x \rightarrow \infty, x \in E_{i}} f(x) & =a_{i} ; \\
\lim _{x \rightarrow \infty, x \in e_{j}} f(x) & =\infty,
\end{aligned}
$$

where $i=1,2, \ldots, l$ and $j \in P$. Conversely, given real numbers $a_{1}, a_{2}$, $\ldots, a_{l} \in \mathbf{R}$ and a subset $P$ of $\{1,2, \ldots, s\}$, there exists nonnegative $\mathcal{A}$ harmonic function $f$ on $N$ satisfying (4) and (5).

Moreover, in the case when $\mathcal{A}$ satisfies (A5) through (A8), each energy finite bounded $\mathcal{A}$-harmonic function $f$ is uniquely determined by the values in (4).

As mentioned above, if a complete Riemannian manifold has nonnegative Ricci curvature outside a compact set and finite first Betti number, then each end of the manifold satisfies the volume doubling condition $(D)_{\infty}$, the Poincaré inequality $(P)_{\infty}$ and the finite covering condition $(\mathrm{FC})$ at infinity. Hence, each end becomes a Harnack end. Therefore, from Corollary 1.2, we have a generalization of results of Yau [20], Donnelly [6], Grigor'yan [7], Li and Tam [15], [16], and Kim and the present author [14] as follows:

COROLlary 1.3. Let $M$ be a complete Riemannian manifolds being roughly isometric to a complete Riemannian manifold with nonnegative Ricci curvature outside a compact set and finite first Betti number. Let $E_{1}, E_{2}, \ldots, E_{l}, l \geq 1$, and $e_{1}, e_{2}, \ldots, e_{s}, s \geq 0$, be $p$-nonparabolic ends and p-parabolic ends of $M$, respectively, and $\mathcal{A}$ be an elliptic operator on $N$ satisfying (A1) through (A5). Then given real numbers $a_{1}, a_{2}, \ldots, a_{l} \in \mathbf{R}$ and a subset $P$ of $\{1,2, \ldots, s\}$, there exists nonnegative $\mathcal{A}$-harmonic function $f$ on $M$ satisfying (4) and (5).

Moreover, in the case when $\mathcal{A}$ satisfies (A5) through (A8), the set of all energy finite bounded $\mathcal{A}$-harmonic functions on $M$ is one to one corresponding to $\mathbf{R}^{l}$. 


\section{$\S 2 . \quad p$-parabolicity and Harnack end}

We begin with defining ends of a complete Riemannian manifold $M$ : Fix a point $o$ in $M$. We denote by $\sharp(r)$ the number of unbounded components of $M \backslash B_{r}(o)$. It is easy to prove that $\sharp(r)$ is nondecreasing in $r>0$. Let $\lim _{r \rightarrow \infty} \sharp(r)=k$, where $k$ may be infinity, then we say that the number of ends of $M$ is $k$. Through this paper, we assume that the number of ends of each manifold is finite. In this case, we can choose $r_{0}>0$ such that $\sharp(r)=k$ for all $r \geq r_{0}$. Hence, there exist mutually disjoint unbounded components $E_{1}, E_{2}, \ldots, E_{k}$ of $M \backslash B_{r_{0}}(o)$ and we call each $E_{i}$ an end of $M$ for $i=1,2, \ldots, k$.

We classify all ends of a complete Riemannian manifold by $p$-parabolicity as follows:

Definition 2.1. We say that an end $E$ of a complete Riemannian manifold $M$ is $p$-nonparabolic if $E$ has positive $p$-capacity at infinity, i.e., for some $r_{1} \geq r_{0}$,

$$
\operatorname{Cap}_{p}\left(E \backslash B_{r_{1}}(o)\right)=\inf _{u} \int_{E \backslash B_{r_{1}}(o)}|\nabla u|^{p}>0
$$

where the infimum is taken over all continuous functions $u$ on $E \backslash B_{r_{1}}(o)$ such that $u$ is compactly supported, smooth on $E \backslash \bar{B}_{r_{1}}(o)$ and $u=1$ on $\partial B_{r_{1}}(o) \cap E$. Otherwise, $E$ is called $p$-parabolic end.

On the other hand, $\mathcal{A}$-harmonic functions are quasi-minimizers of $p$ Dirichlet integral, i.e., if $u \in W^{1, p}(\Omega)$ is a solution of (1) and $u-\phi \in$ $W_{0}^{1, p}(\Omega)$, then there exists a constant $C<\infty$ such that

$$
\int_{\Omega}|\nabla u|^{p} \leq C \int_{\Omega}|\nabla \phi|^{p}
$$

Therefore, the $p$-parabolicity of an end $E$ is equivalent to the existence of a continuous function $u_{E}$, called an $\mathcal{A}$-harmonic measure of $E$, on $E$ such that

$$
\left\{\begin{array}{cll}
\mathcal{A} u_{E}=0 & \text { in } & E \backslash \bar{B}_{r_{1}}(o) ; \\
u_{E}=0 & \text { on } & B_{r_{1}}(o) \cap E ; \\
\sup _{E \backslash B_{r_{1}}(o)} u_{E}=1 . & &
\end{array}\right.
$$

We now give a characterization of $p$-parabolic ends as follows: 
LEMMA 2.2. Let e be a p-parabolic end of a complete Riemannian manifold and $f$ be a nonconstant $\mathcal{A}$-harmonic function bounded below on $e$. Then

$$
\inf _{e} f<\liminf _{x \rightarrow \infty, x \in e} f(x) .
$$

Proof. Suppose that $\liminf _{x \rightarrow \infty, x \in e} f(x)=\inf _{e} f=m>-\infty$. Since $f$ is nonconstant, there exists a proper open subset $\Omega$ of $e$ such that $\Omega=\{x \in$ $e: f(x)<m+\epsilon\}$ for sufficiently small $\epsilon>0$. Put $v=\max \{(m+\epsilon-f) / \epsilon, 0\}$. Then $v$ is a nonnegative subsolution for the operator $\mathcal{A}$ on $e$ such that $v \equiv 0$ on $e \backslash \Omega$ and $\sup _{e} v=1$. By the comparison principle, we can construct an $\mathcal{A}$-harmonic measure $u_{e}$ of $e$, which contradicts $p$-parabolicity of $e$.

We now introduce a nonnegative $\mathcal{A}$-harmonic function in each $p$-parabolic end $e$, which virtually takes over the role as a barrier at infinity of $e$ in Section 3. (See [11] for the proof.)

Lemma 2.3. Let e be a p-parabolic end. Then there exists a nonnegative $\mathcal{A}$-harmonic function $v_{e}$ in e such that $v_{e}=0$ on $\partial B_{r_{0}}(o) \cap E$ and $v_{e}$ is unbounded on $e$.

Let $o$ be a fixed point of a complete Riemannian manifold $M$. We say that an end $E$ of $M$ is Harnack if there exist a sequence $\left\{H_{R}\right\}$ of compact (not necessarily connected) subsets of $E$ and a constant $C<\infty$ such that for any nonnegative $\mathcal{A}$-harmonic function $f$ in $E$,

$$
\sup _{H_{R}} f \leq C \inf _{H_{R}} f
$$

where $d\left(o, H_{R}\right) \rightarrow \infty$ as $R \rightarrow \infty$, and each $H_{R}$ divides $E$ into a bounded subset and the unbounded component of $E \backslash H_{R}$.

Let us consider some examples of Harnack ends.

ExAMPLE 2.4. (i) Let $E$ be an end of a complete Riemannian manifold $M$ and $o$ be fixed point of $M$. Suppose that $E$ satisfies the volume doubling condition and the Poincaré inequality as follows:

$(D)_{\infty}$ for given $0<\alpha<1 / 2$, there is a constant $C<\infty$ depending only on $\alpha$ such that for any point $x \in \partial B_{R}(o) \cap E$ and any $0<r<R / 2$,

$$
\operatorname{vol} B_{r}(x) \leq C \operatorname{vol} B_{\alpha r}(x)
$$


$(P)_{\infty}$ there exist a constant $C<\infty$ and an integer $k \in \mathbf{N}$ such that for any point $x \in \partial B_{R}(o) \cap E$, any $0<r<R / 2$ and all $f \in C^{\infty}\left(B_{r}(x)\right)$,

$$
\int_{B_{r / k}(x)}|f-\bar{f}| \leq C r\left(\operatorname{vol} B_{r}(x)\right)^{1-1 / p}\left(\int_{B_{r}(x)}|\nabla f|^{p}\right)^{1 / p}
$$

where $\bar{f}=\left(\operatorname{vol} B_{r / k}(x)\right)^{-1} \int_{B_{r / k}(x)} f$.

By the result of [11], the Harnack inequality is valid on each ball $B_{r}(x) \subset E$ with $0<r<d(o, x) / 2$, i.e., there exists a constant $C<\infty$ such that for any nonnegative $\mathcal{A}$-harmonic function $f$ in $B_{r}(x)$,

$$
\sup _{B_{r / 2}(x)} f \leq C \inf _{B_{r / 2}(x)} f .
$$

We now add the following finite covering condition on $E$ :

(FC) for given $0<\alpha<1 / 4$, there exist a positive integer $m=m(\alpha)$ and points $x_{1}, x_{2}, \ldots, x_{m}$ in $\partial C_{E, R}$ such that $\bigcup_{i=1}^{m} B_{\alpha R}\left(x_{i}\right)$ is connected and $\partial C_{E, R} \subset \bigcup_{i=1}^{m} B_{\alpha R}\left(x_{i}\right)$, where $C_{E, R}$ denotes the unbounded component of $E \backslash B_{R}(o)$.

This condition gives a uniform connectedness near infinity of $E$. Therefore, we get the Harnack inequality as follows: There exists a constant $C<\infty$ such that for any nonnegative $\mathcal{A}$-harmonic function $f$ in $E$,

$$
\sup _{\partial C_{E, R}} f \leq C \inf _{\partial C_{E, R}} f .
$$

(ii) Let $M$ be a complete Riemannian manifold with nonnegative Ricci curvature outside a compact set. Then the volume doubling condition $(D)_{\infty}$ and the Poincaré inequality $(P)_{\infty}$ are satisfied on each end of $M$. In addition, if the manifold $M$ has finite first Betti number, then the finite covering condition (FC) also holds on each end. (See [16] and [17].)

(iii) As the simplest case, let $M$ be a complete Riemannian manifold with nonnegative Ricci curvature everywhere. Then by the splitting theorem of Cheeger and Gromoll [4], $M$ has at most two ends. Furthermore, for each end $E$ of $M, \partial B_{R}(o) \cap E$ is connected, where $o$ is a fixed point of $M$. (See [1].) Since $M$ satisfies the volume doubling condition globally, the finite covering condition (FC) is valid on each end. The Poincaré inequality also holds globally on $M$. Therefore, every end of a connected sum of complete Riemannian manifolds with nonnegative Ricci curvature is Harnack. 
Let us end this section by mentioning the rough isometric invariance of $(D)_{\infty},(P)_{\infty},(\mathrm{FC})$ and the $p$-parabolicity of ends. In fact, we can prove that if an end $E$ is roughly isometric to an end satisfying $(D)_{\infty},(P)_{\infty}$ and (FC), then $E$ is also a Harnack end. Therefore, using this result, one can find further examples of Harnack ends.

A rough isometry $\varphi: X \rightarrow Y$ is a (not necessarily continuous) map between two metric spaces $X$ and $Y$ satisfying the following conditions:

(R1) for some $\tau>0$, the $\tau$-neighborhood of the image $\varphi(X)$ covers $Y$;

(R2) there exist constants $a \geq 1$ and $b \geq 0$ such that

$$
\frac{1}{a} d\left(x_{1}, x_{2}\right)-b \leq d\left(\varphi\left(x_{1}\right), \varphi\left(x_{2}\right)\right) \leq a d\left(x_{1}, x_{2}\right)+b
$$

for all $x_{1}, x_{2} \in X$, where $d$ denotes the distances of $X$ and $Y$ induced from their metrics, respectively.

Especially, being roughly isometric is an equivalence relation. (See [12] or [5].) However, even if two metric spaces are roughly isometric to each other, their topologies may be completely different, since the rough isometry is not assumed to be continuous. So, in order to deploy our theory via rough isometry, we need to add the following local conditions on manifolds: Let $\varphi: M \rightarrow N$ be a rough isometry between manifolds $M$ and $N$.

$(D)_{\text {loc }}$ there exists a constant $C_{r}<\infty$ depending only on $r>0$ such that for any point $x$ in $M$ (in $N$, respectively)

$$
\operatorname{vol} B_{2 r}(x) \leq C_{r} \operatorname{vol} B_{r}(x)
$$

$(P)_{\text {loc }}$ there exists a constant $C_{r}<\infty$ depending only on $r>0$ such that for any point $x$ in $M$ (in $N$, respectively) and for all $f \in C^{\infty}\left(B_{r}(x)\right)$

$$
\int_{B_{r}(x)}|f-\bar{f}| \leq C_{r} \int_{B_{r}(x)}|\nabla f|,
$$

where $\bar{f}=\left(\operatorname{vol} B_{r}(x)\right)^{-1} \int_{B_{r}(x)} f$;

$(C)_{\text {loc }}$ there exists a constant $C \geq 1$ such that for any point $x$ in $M$

$$
\frac{1}{C} \operatorname{vol} B_{1}(x) \leq \operatorname{vol} B_{1}(\varphi(x)) \leq C \operatorname{vol} B_{1}(x) .
$$


Note that these local assumptions are satisfied on any complete Riemannian manifold with the Ricci curvature bounded below by a constant and the positive injectivity radius. (See [3] or [12].) From now on, when we say that a map $\varphi: M \rightarrow N$ is a rough isometry between complete Riemannian manifolds $M$ and $N$, it means that the map $\varphi$ satisfies the conditions (R1), (R2) and $(C)_{\text {loc }}$, and $M$ and $N$ satisfy the local conditions $(D)_{\text {loc }}$ and $(P)_{\text {loc }}$, unless otherwise specified.

In [14], Kim and the present author proved that the number of ends of complete Riemannian manifolds is preserved under rough isometries between complete Riemannian manifolds, moreover, the restriction map to each end becomes a rough isometry between ends. On the other hand, slightly modifying the program of Kanai [11], [12], one can obtain the rough isometric invariance of the conditions $(D)_{\infty}$ and $p$-parabolicity of ends. Recently, Coulhon and Saloff-Coste [5] proved that the Poincaré inequality is invariant under rough isometries between complete Riemannian manifolds. One can easily apply this result to our situation. Now we have only to check the invariance of the finite covering condition (FC). However, we get only the modified version of the finite covering condition via rough isometry. Nonetheless, this modified one still gives a uniform connectedness near infinity as follows:

Lemma 2.5. Let $\varphi: D \rightarrow E$ be a rough isometry between ends of complete Riemannian manifolds. Suppose that $D$ satisfies the finite covering condition (FC). Then there exist a sequence $\left\{H_{R}\right\}$ of hypersurfaces in $E$ such that $d\left(o, H_{R}\right) \rightarrow \infty$ as $R \rightarrow \infty$ for a fixed point $o \in E$ and each $H_{R}$ divides $E$ into a bounded subset and the unbounded component of $E \backslash H_{R}$, and a positive integer $\tilde{m}$ and finitely many points $y_{1}, y_{2}, \ldots, y_{\tilde{m}}$ such that $\bigcup_{i=1}^{\tilde{m}} B_{\beta R}\left(y_{i}\right)$ is connected and $H_{R} \subset \bigcup_{i=1}^{\tilde{m}} B_{\beta R}\left(y_{i}\right)$, where $0<\beta<1 / 4$ is independent of $R$.

Proof. Fix a point $\tilde{o}$ in $D$ and put $o=\varphi(\tilde{o})$. Choose a smallest integer $n \in \mathbf{N}$ such that $n \geq 8 a^{2} / \alpha$ and a finite sequence $\left\{R_{j}\right\}_{j=0,1, \ldots, n}$ such that $R_{0}=R / 2 a$ and $R_{j}=R_{j-1}+\alpha R / 4 a$ for $j=1,2, \ldots, n$. Then by (FC), there exist an integer $l \in \mathbf{N}$ and points $x_{1}^{j}, x_{2}^{j}, \ldots, x_{l}^{j} \in \partial C_{D, R_{j}}$ such that

$$
\left(B_{a(R+\tau+b)}(\tilde{o}) \backslash \bar{B}_{(R-\tau-b) / a}(\tilde{o})\right) \cap D \subset \bigcup_{j=0}^{n} \bigcup_{i=1}^{l} B_{a \alpha R}\left(x_{i}^{j}\right) \cup \bigcup_{j=0}^{n} A_{j}
$$


and $\bigcup_{j=0}^{n} \bigcup_{i=1}^{l} B_{a \alpha R}\left(x_{i}^{j}\right)$ is connected, where $A_{j}$ is the union of bounded components of $D \backslash B_{R_{j}}(\tilde{o})$. Also by (R2), $\bigcup_{j=0}^{n} \bigcup_{i=1}^{l} B_{\beta R}\left(\varphi\left(x_{i}^{j}\right)\right)$ is connected, where $\beta=3 a^{2} \alpha$.

We claim that $\bigcup_{j=0}^{n} \bigcup_{i=1}^{l} B_{\beta R}\left(\varphi\left(x_{i}^{j}\right)\right)$ divides $E$ into a bounded subset and the unbounded component of $E \backslash \bigcup_{j=0}^{n} \bigcup_{i=1}^{l} B_{\beta R}\left(\varphi\left(x_{i}^{j}\right)\right)$. Otherwise, there exist a point $y \in \partial C_{E, R} \backslash\left(\bigcup_{j=0}^{n} \bigcup_{i=1}^{l} B_{3 a^{2} \alpha R}\left(\varphi\left(x_{i}^{j}\right)\right)\right)$ and an arclength parameterized curve $\gamma:[0, \infty) \rightarrow E \backslash B_{R}(o)$ such that $\gamma(0)=y, d(o, \gamma(t)) \rightarrow$ $\infty$ as $t \rightarrow \infty$ and

$$
B_{c}(\gamma[0, \infty)) \cap\left(\bigcup_{j=0}^{n} \bigcup_{i=1}^{l} B_{2 a^{2} \alpha R}\left(\varphi\left(x_{i}^{j}\right)\right)\right)=\emptyset
$$

for some $c>5 a^{2}(\tau+a+b)$. From the condition (R1), we can choose a sequence $\left\{z_{k}\right\}_{k \in \mathbf{N}}$ in $D \backslash B_{R / 2 a}(\tilde{o})$ such that $d\left(\varphi\left(z_{k}\right), \gamma(k)\right) \leq \tau$ for each $k \in \mathbf{N}$. Therefore, there exists a curve $\sigma:[0, \infty) \rightarrow D \backslash B_{R / 2 a}(\tilde{o})$ such that $\sigma(0) \in \partial B_{R / 2 a}(\tilde{o}) \cap D, d(\tilde{o}, \sigma(t)) \rightarrow \infty$ as $t \rightarrow \infty$ and $\sigma[0, \infty) \subset$ $\bigcup_{k=0}^{\infty} B_{a(2 \tau+b+1)}\left(z_{k}\right)$. By $(\mathrm{R} 2), \varphi(\sigma[0, \infty)) \subset B_{4 a^{2}(\tau+a+b)}(\gamma[0, \infty))$ and this implies that

$$
B_{\tau}(\varphi(\sigma[0, \infty))) \subset B_{c}(\gamma[0, \infty))
$$

On the other hand, since $\sigma(0) \in B_{2 a R}(\tilde{o})$ and $\sigma(t) \rightarrow \infty$ as $t \rightarrow \infty$, there exists a point $x_{0} \in \sigma[0, \infty) \cap \partial C_{D, 2 a R}$. Hence by $(8), \varphi\left(x_{0}\right) \in B_{c}(\gamma[0, \infty))$. However since

$$
\varphi\left(\partial C_{D, 2 a R}\right) \subset B_{\tau}\left(\varphi\left(\bigcup_{j=0}^{n} \bigcup_{i=1}^{l} B_{\alpha R}\left(x_{i}^{j}\right)\right)\right) \subset \bigcup_{j=0}^{n} \bigcup_{i=1}^{l} B_{2 a^{2} \alpha R}\left(\varphi\left(x_{i}^{j}\right)\right),
$$

this contradicts $(7)$.

\section{$\S 3$. Proof of main results}

Any nonnegative $\mathcal{A}$-harmonic function on a Harnack end satisfies the asymptotically constant property as follows: (See [11] for the proof.)

LEMMA 3.1. Let $E$ be a Harnack end and $f$ be a nonnegative $\mathcal{A}$ harmonic function on $E$. Then there exists a constant $0 \leq C_{f} \leq \infty$ such that

$$
\lim _{x \rightarrow \infty, x \in E} f(x)=C_{f}
$$

In particular, if $E$ is p-nonparabolic, then $f$ is bounded at infinity, hence the limit value $C_{f}$ in (9) is finite. 
We construct nonnegative $\mathcal{A}$-harmonic functions corresponding to each $p$-parabolic end, in particular, each of which takes the infinity as the value at infinity of each corresponding $p$-parabolic end.

LEMMA 3.2. Let $M$ have at least one p-nonparabolic end, and e be a p-parabolic and Harnack end of $M$. Then there exists a nonnegative $\mathcal{A}$ harmonic function $h$ on $M$ such that

(i) $\lim _{x \rightarrow \infty, x \in e} h(x)=\infty$;

(ii) $\lim _{x \rightarrow \infty, x \in E} h(x)=0$ for any $p$-nonparabolic end $E$;

(iii) $0 \leq h \leq C_{h}$ on $M \backslash e$, where $C_{h}$ is a constant.

Proof. By Lemma 2.3 and Lemma 3.1, there exists a nonnegative continuous function $v_{e}$ on $e$ such that $v_{e}$ is $\mathcal{A}$-harmonic in $e, v_{e}=0$ on $\partial B_{r_{0}}(o) \cap e$ and $\lim _{x \rightarrow \infty, x \in e} v_{e}(x)=\infty$. Let $\left\{h_{r}\right\}_{r>r_{0}}$ be a sequence of continuous functions on $\bar{B}_{r}(o)$ such that $h_{r}$ is $\mathcal{A}$-harmonic in $B_{r}(o), h_{r}=v_{e}$ on $\partial B_{r}(o) \cap e$ and $h_{r}=0$ on $\partial B_{r}(o) \backslash e$.

We claim that $\left\{h_{r}\right\}$ is uniformly bounded on $\partial B_{r_{0}}(o) \cap e$. Otherwise, there exists an increasing sequence $\left\{r_{i}\right\}$ such that $a_{r_{i}}=\max _{\partial B_{r_{0}}(o) \cap e} h_{r_{i}} \rightarrow$ $\infty$ as $r_{i} \rightarrow \infty$. Put $H_{i}=h_{r_{i}} / a_{r_{i}}$, then $H_{i}=v_{e} / a_{r_{i}}$ on $\partial B_{r_{i}}(o) \cap e, H_{i}=0$ on $\partial B_{r_{i}}(o) \backslash e$ and $\max _{\partial B_{r_{0}}(o) \cap e} H_{i}=1$. By the comparison principle, we get

$$
0 \leq H_{i} \leq v_{e} / a_{r_{i}}+1 \text { on } B_{r_{i}}(o) \cap e .
$$

Since $0 \leq H_{i} \leq 1$ on $B_{r_{i}}(o) \backslash e$, the sequence $\left\{H_{i}\right\}$ is locally uniformly bounded. By the result of [8], the sequence $\left\{H_{i}\right\}$ is equicontinuous. Hence by Ascoli's theorem, there exists a subsequence converging uniformly to an $\mathcal{A}$-harmonic function $H$ on any compact subset of $M$ such that $0 \leq H \leq 1$ on $M$.

On the other hand, since $0 \leq H_{i} \leq 1$ on $B_{r_{i}}(o) \backslash\left(e \backslash B_{r_{0}}(o)\right)$, for each $p$-nonparabolic end $E, 0 \leq H_{i} \leq 1-u_{E}$ on $B_{r}(o) \cap E$, where $u_{E}$ is the $\mathcal{A}$-harmonic measure given in (6). Hence we get

$$
\lim _{x \rightarrow \infty, x \in E} H(x)=0 .
$$

Since $\max _{\partial B_{r_{0}}(o) \cap e} H_{i}=1$, we can suitably choose a subsequence of $\left\{H_{i}\right\}$ in such a way that $\max _{\partial B_{r_{0}}(o) \cap e} H=1$. Then by the strong maximum principle, $H \equiv 1$, but this contradicts (10). Therefore, $\left\{h_{r}\right\}$ is uniformly bounded on $\partial B_{r_{0}}(o) \cap e$. 
Put $\bar{a}=\sup _{r} a_{r}$. Then by the comparison principle, $v_{e} \leq h_{r_{i}} \leq v_{e}+\bar{a}$ on $\left(B_{r_{i}}(o) \backslash B_{r_{0}}(o)\right) \cap e$. By Ascoli's theorem, there exists a subsequence, denoted again by $\left\{h_{r_{i}}\right\}$, converging uniformly on any compact subset of $M$. The limit function $h=\lim _{i \rightarrow \infty} h_{r_{i}}$ is a nonnegative $\mathcal{A}$-harmonic function on $M$ such that

$$
v_{e} \leq h \leq v_{e}+\bar{a} \text { on } e \backslash B_{r_{0}}(o),
$$

hence we get (i). On the other hand, since $h_{r}=0$ on $\partial B_{r}(o) \backslash e$ and $h_{r} \leq \bar{a}$ on $\partial B_{r_{0}}(o) \cap e$, by the comparison principle, we have $0 \leq h_{r} \leq \bar{a}\left(1-u_{E}\right)$ on $B_{r}(o) \cap E$ for each $p$-nonparabolic end $E$ and $0 \leq h_{r} \leq \bar{a}$ on $B_{r}(o) \backslash(e \backslash$ $\left.B_{r_{0}}(o)\right)$. Hence we get

$$
0 \leq h \leq \bar{a}\left(1-u_{E}\right) \text { on } E \text { and } 0 \leq h \leq \bar{a} \text { on } M \backslash\left(e \backslash B_{r_{0}}(o)\right)
$$

These imply (ii) and (iii), respectively.

Proof of Theorem 1.1. From Lemma 3.2, we can choose a nonnegative $\mathcal{A}$-harmonic function $h_{j}$ on $M$ for each $j \in P$ such that

(i) $\lim _{x \rightarrow \infty, x \in e_{j}} h_{j}(x)=\infty$;

(ii) $\lim _{x \rightarrow \infty, x \in E_{i}} h_{j}(x)=0$ for each $i=1,2, \ldots, l$;

(iii) $0 \leq h_{j} \leq C_{j}<\infty$ on $M \backslash e_{j}$.

Put $C=\max \left\{\sup _{M \backslash e_{j}} h_{j}: j \in P\right\}$. Without loss of generality, we may assume that $0<a_{1} \leq a_{2} \leq \ldots \leq a_{l} \leq 2 a_{1}$ and $C \leq 2 a_{1}-a_{l}$. By the comparison principle, we have $h_{j}+a_{i} \leq a_{i}\left(2-u_{E_{i}}\right)$ on each $E_{i}$, where $u_{E_{i}}$ is the $\mathcal{A}$-harmonic measure of $E_{i}$. Now construct a sequence $\left\{f_{r}\right\}_{r>r_{0}}$ of continuous functions such that

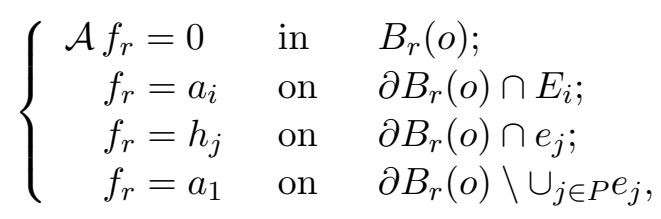

where $i=1,2, \ldots, l$ and $j \in P$. By the comparison principle, $f_{r} \leq h_{j}+a_{i}$ on $B_{r}(o) \cap E_{i}$, hence $a_{i} u_{E_{i}} \leq f_{r} \leq a_{i}\left(2-u_{E_{i}}\right)$ on $B_{r}(o) \cap E_{i}$. On the other hand, $h_{j} \leq f_{r} \leq h_{j}+a_{l}$ on $B_{r}(o) \cap e_{j}$.

By Ascoli's theorem, there exists a subsequence, denoted again by $\left\{f_{r}\right\}$, converging uniformly on any compact subset of $M$. The limit function $f=$ $\lim _{r \rightarrow \infty} f_{r}$ is a nonnegative $\mathcal{A}$-harmonic function on $M$ such that $a_{i} u_{E_{i}} \leq$ 
$f \leq a_{i}\left(2-u_{E_{i}}\right)$ on $E_{i}$ for each $i=1,2, \ldots, l, h_{j} \leq f \leq h_{j}+a_{l}$ on $e_{j}$ for each $j \in P$. Hence we get

$$
\lim _{x \rightarrow \infty, x \in E_{i}} f(x)=a_{i} \text { and } \lim _{x \rightarrow \infty, x \in e_{j}} f(x)=\infty
$$

where $i=1,2, \ldots, l$ and $j \in P$. On the other hand, since $f_{r} \leq \max \left\{h_{j}: j \in\right.$ $P\}+a_{l}$ on $B_{r}(o) \backslash \cup_{j \in P} e_{j}, f$ is bounded on $M \backslash \cup_{j \in P} e_{j}$.

Note that in the case that $P=\emptyset$, we get the consequence by neglecting the role of the $\mathcal{A}$-harmonic function $h_{j}$ corresponding to $p$-parabolic end $e_{j}$.

Let us prove the last statement. Suppose that $f$ and $f^{\prime}$ are energy finite bounded $\mathcal{A}$-harmonic function on $M$ such that $\lim _{x \rightarrow \infty, x \in E}\left(f-f^{\prime}\right)(x)=0$ for each $p$-nonparabolic end $E$ of $M$. Put $g=\max \left\{f-f^{\prime}, 0\right\}$ and $C_{g}=$ $\sup _{M} g$. Since $\lim _{x \rightarrow \infty, x \in E} g(x)=0$ for any $p$-nonparabolic end $E$ of $M$, for given $\epsilon>0$, there exists $R_{0}$ such that $g(x) \leq \epsilon$ for all $x \in E \backslash B_{R}(o)$, whenever $R \geq R_{0}$.

Choose a sequence $\left\{h_{R}\right\}$ of continuous functions such that $h_{R}$ is $\mathcal{A}$ harmonic in $B_{R}(o)$ and $h_{R}=g$ on $\partial B_{R}(o)$. Then for sufficiently large $R \geq R_{0}, h_{R} \leq C_{g}\left(1-u_{E}\right)+\epsilon$ on $\partial B_{R}(o) \cap E$ and $h_{R} \leq C_{g}\left(1-u_{E}\right)$ on $\partial B_{r_{1}}(o) \cap E$, where $u_{E}$ is the $\mathcal{A}$-harmonic measure of $E$. By the comparison principle,

$$
0 \leq h_{R} \leq C_{g}\left(1-u_{E}\right)+\epsilon \text { on } B_{R}(o) \cap E
$$

By Ascoli's theorem, there exists a subsequence $\left\{h_{R_{n}}\right\}$ converging uniformly to an $\mathcal{A}$-harmonic function $h$ on any compact subset of $M$. Moreover, by the minimizing property of $\mathcal{A}$-harmonic function, $h$ has the finite energy.

Put $\phi_{n}=g-h_{n}$ with $h_{n}=h_{R_{n}}$, then each $\phi_{n}$ has a compact support and $\left\{\phi_{n}\right\}$ converges uniformly to $\phi=g-h$ on any compact subset of $M$. We will prove that

$$
\lim _{n \rightarrow \infty} \mathbf{J}\left(\phi-\phi_{n}, M\right)=0 .
$$

On the other hand, there exists a constant $\alpha<\infty$ such that

$$
\alpha=\inf _{\eta} \mathbf{J}(g-\eta, M)
$$

where the infimum is taken over all compactly supported smooth functions $\eta$ on $M$. By the minimizing property of $\mathcal{A}$-harmonic functions,

$$
\alpha=\lim _{n \rightarrow \infty} \mathbf{J}\left(h_{n}, M\right)
$$


In case $2 \leq p<\infty$, by (A8),

$$
\begin{aligned}
\alpha & \leq \mathbf{J}\left(\left(h_{n}+h_{m}\right) / 2, M\right) \\
& \leq \mathbf{J}\left(\left(h_{n}+h_{m}\right) / 2, M\right)+\mathbf{J}\left(\left(h_{n}-h_{m}\right) / 2, M\right) \\
& \leq 2^{-1}\left(\mathbf{J}\left(h_{n}, M\right)+\mathbf{J}\left(h_{m}, M\right)\right) \rightarrow \alpha \text { as } n, m \rightarrow \infty,
\end{aligned}
$$

and in case $1<p \leq 2$, by (A8),

$$
\begin{aligned}
\alpha^{p^{\prime}} & \leq \mathbf{J}\left(\left(h_{n}+h_{m}\right) / 2, M\right)^{p^{\prime}} \\
& \leq \mathbf{J}\left(\left(h_{n}+h_{m}\right) / 2, M\right)^{p^{\prime}}+\mathbf{J}\left(\left(h_{n}-h_{m}\right) / 2, M\right)^{p^{\prime}} \\
& \leq\left(2^{-1}\left(\mathbf{J}\left(h_{n}, M\right)+\mathbf{J}\left(h_{m}, M\right)\right)\right)^{p^{\prime}} \rightarrow \alpha^{p^{\prime}} \text { as } n, m \rightarrow \infty,
\end{aligned}
$$

where $p^{\prime}=1 /(p-1)$. Therefore, we get

$$
\mathbf{J}\left(h_{n}-h_{m}\right)=0 \text { as } n, m \rightarrow \infty .
$$

By the Lebesgue dominated convergence theorem, we have

$$
\lim _{n \rightarrow \infty} \mathbf{J}\left(h-h_{n}\right)=0,
$$

which is equivalent to (11).

Note again that $0 \leq h \leq C_{g}\left(1-u_{E}\right)+\epsilon$ on each $p$-nonparabolic end $E$. Since $\lim _{x \rightarrow \infty, x \in E} u_{E}(x)=1$ and $\epsilon>0$ is chosen arbitrarily, we have $\lim _{x \rightarrow \infty, x \in E} h(x)=0$ for each $p$-nonparabolic end $E$ of $M$. Hence, by Lemma $2.2, h \equiv 0$ on $M$.

Applying the above argument to $w=\max \left\{f^{\prime}-f, 0\right\}$, we can choose a sequence $\left\{\psi_{n}\right\}$ of compactly supported continuous functions such that

$$
\lim _{n \rightarrow \infty} \mathbf{J}\left(w-\psi_{n}, M\right)=0 .
$$

Combining (11) and (12), the sequence $\left\{\phi_{n}-\psi_{n}\right\}$ of compactly supported continuous functions such that

$$
\lim _{n \rightarrow \infty} \mathbf{J}\left(\left(f-f^{\prime}\right)-\left(\phi_{n}-\psi_{n}\right), M\right)=0 .
$$

By (A3) and the Hölder inequality, we get

$$
\begin{aligned}
& \int_{M}\left\langle\mathcal{A}(\nabla f), \nabla\left(f-f^{\prime}\right)-\nabla\left(\phi_{n}-\psi_{n}\right)\right\rangle \\
\leq & C_{2} \int_{M}|\nabla f|^{p-1}\left|\nabla\left(\left(f-f^{\prime}\right)-\left(\phi_{n}-\psi_{n}\right)\right)\right| \\
\leq & C_{2}\left(\int_{M}|\nabla f|^{p}\right)^{(p-1) / p}\left(\int_{M}\left|\nabla\left(\left(f-f^{\prime}\right)-\left(\phi_{n}-\psi_{n}\right)\right)\right|^{p}\right)^{1 / p} .
\end{aligned}
$$


Using (A7), (13) and the energy finiteness of $f$, we have

$$
\int_{M}\left\langle\mathcal{A}(\nabla f), \nabla\left(f-f^{\prime}\right)-\nabla\left(\phi_{n}-\psi_{n}\right)\right\rangle \rightarrow 0 \text { as } n \rightarrow \infty .
$$

Since $f$ is an $\mathcal{A}$-harmonic function, this implies that

$$
\int_{M}\left\langle\mathcal{A}(\nabla f), \nabla\left(f-f^{\prime}\right)\right\rangle=0 .
$$

Similarly arguing, we have

$$
\int_{M}\left\langle\mathcal{A}\left(\nabla f^{\prime}\right), \nabla\left(f-f^{\prime}\right)\right\rangle=0 .
$$

These imply the following identities;

$$
\int_{M}\langle\mathcal{A}(\nabla f), \nabla f\rangle=\int_{M}\left\langle\mathcal{A}(\nabla f), \nabla f^{\prime}\right\rangle
$$

and

$$
\int_{M}\left\langle\mathcal{A}\left(\nabla f^{\prime}\right), \nabla f^{\prime}\right\rangle=\int_{M}\left\langle\mathcal{A}\left(\nabla f^{\prime}\right), \nabla f\right\rangle .
$$

Using these identity,

$$
\begin{aligned}
& \int_{M}\left\langle\mathcal{A}(\nabla f)-\mathcal{A}_{x}\left(\nabla f^{\prime}\right), \nabla f-\nabla f^{\prime}\right\rangle \\
= & \int_{M}\langle\mathcal{A}(\nabla f), \nabla f\rangle-\int_{M}\left\langle\mathcal{A}(\nabla f), \nabla f^{\prime}\right\rangle-\int_{M}\left\langle\mathcal{A}\left(\nabla f^{\prime}\right), \nabla f\right\rangle \\
+ & +\int_{M}\left\langle\mathcal{A}\left(\nabla f^{\prime}\right), \nabla f^{\prime}\right\rangle \\
= & 0 .
\end{aligned}
$$

Thus by (A4), $f-f^{\prime} \equiv C$ for a constant $C$. Since $\lim _{x \rightarrow \infty, x \in E}\left(f-f^{\prime}\right)(x)=0$ for each $p$-nonparabolic end $E$ of $M$, we have $f \equiv f^{\prime}$.

As mentioned in Section 2, if an end $E$ is roughly isometric to an end satisfying the conditions $(D)_{\infty},(P)_{\infty}$ and $(\mathrm{FC})$, then $E$ becomes a Harnack end. Also since these conditions are satisfied on each end of a complete Riemannian manifolds with nonnegative Ricci curvature outside a compact subset and finite first Betti number, we have Corollary 1.2 and Corollary 1.3 . 
By the result of [11], when $M$ is a complete Riemannian manifold with nonnegative Ricci curvature, the $p$-nonparabolicity of $M$ is equivalent to

$$
\int_{r_{0}}^{\infty}\left(\frac{r}{\operatorname{vol} B_{r}(o)}\right)^{1 /(p-1)} d r<\infty
$$

for a fixed point $o$ in $M$ and some $r_{0}>0$. On the other hand, by the splitting theorem [4], $M$ has at most two ends. Furthermore, if $M$ has two ends, then $M$ is isometric to $K \times \mathbf{R}$, where $K$ is compact, hence $M$ is $p$ parabolic. Therefore, applying our result to this simple situation, we have the following corollary:

Corollary 3.3. Let $M_{1}, M_{2}, \ldots, M_{k}$ be complete Riemannian manifolds with nonnegative Ricci curvature and $M$ be a complete Riemannian manifold being roughly isometric to a connected sum of $M_{1}, M_{2}, \ldots, M_{k}$. Then the set of all energy finite bounded $\mathcal{A}$-harmonic functions on $M$ is one to one corresponding to $\mathbf{R}^{l}$, where $l$ is the number of $M_{i}$ 's satisfying (14).

\section{REFERENCES}

[1] U. Abresch and D. Gromoll, On complete manifolds with nonnegative Ricci curvature, J. Amer. Math. Soc., 6 (1990), 355-374.

[2] R. Bishop and R. Crittenden, Geometry of manifolds, 2, Academic Press, New York and London, 1964.

[3] P. Buser, A note on the isoperimetric constant, Ann. Sci. Ec. Norm. Sup. Paris, 15 (1982), 213-230.

[4] J. Cheeger and D. Gromoll, The splitting theorem for manifolds of nonnegative Ricci curvature, J. Differential Geom., 6 (1971), 119-128.

[5] Th. Coulhon and L. Saloff-Coste, Variétés riemanniennes isométriques á l'infini, Rev. Mat. Iberoamericana, 11 (1995), 687-726.

[6] H. Donnelly, Bounded harmonic functions and positive Ricci curvature, Math. Z., 191 (1986), 559-565.

[7] A. Grigor'yan, Set of positive solutions of Laplace-Beltrami equation on special type of Riemannian manifolds, Izv. Vyssh. Uchebn. Zaved., Matematika: English transl. Soviet Math., 31 (1987), 48-60.

[8] J. Heinonen, T. Kilpeläinen and O. Martio, Nonlinear potential theory of degenerate elliptic equations, Oxford University Press, Oxford, New York, Tokyo, 1993.

[9] E. Hewitt and K. Stomberg, Real and Abstract Analysis, Springer-Verlag, New York, Heidelberg, Berlin, 1965.

[10] I. Holopainen, Solutions of elliptic equations on manifolds with roughly Euclidean ends, Math. Z., 217 (1994), 459-477. 
[11] - Volume growth, Green's functions and parabolicity of ends, Duke Math. J., 97 No. 2 (1999), 319-346.

[12] M. Kanai, Rough isometries, and combinatorial approximations of geometries of non-compact riemannian manifolds, J. Math. Soc. Japan, 37 No. 3 (1985), 391-413.

[13] L Rough isometries and the parabolicity of riemannian manifolds, J. Math. Soc. Japan, 38 No. 2 (1986), 227-238.

[14] S. W. Kim and Y. H. Lee, Generalized Liouville property for Schrödinger operator on Riemannian manifolds, Math. Z. (To appear).

[15] P. Li and L. F. Tam, Positive harmonic functions on complete Riemannian manifolds with non-negative curvature outside a compact set, Ann. of Math., 125 (1987), 171-207.

[16] _ Green's functions, harmonic functions, and volume comparison, J. Differential Geom., 41 (1995), 277-318.

[17] Z-D. Liu, Ball Covering Property and Nonnegative Ricci curvature Outside a Compact Set, Proc. Symp. Pure Math., 54 (1993), 459-464.

[18] J. Malý and W. P. Ziemer, Fine Regularity of Solutions of Elliptic Partial Differential Equations, 51, Amer. Math. Soc., Mathematical Surveys and Monographs, 1997.

[19] L. Saloff-Coste, Uniformly elliptic operators on Riemannian manifolds, J. Differential Geom., 36 (1992), 417-450.

[20] S. T. Yau, Harmonic functions on complete Riemannian manifolds, Comm. Pure Appl. Math., 28 (1975), 201-228.

Department of Mathematics Education

Ewha Women's University

Seoul 120-750

Korea

yonghah@ewha.ac.kr 